\title{
Lebensgemeinschaft oder Zwangsehe?
}

Laura Bertini im Interview mit Stefan Spring (StS)

Es werden neue Technologien angeboten, um den Alltag zu erleichtern und sicherer zu machen. Jedoch entwickeln sind die Dinge manchmal anders als beabsichtigt. Laura Bertini hat die Hindernisse im Zusammenhang mit einem Projekt in der italienischsprachigen Schweiz analysiert.

\begin{abstract}
StS: Die SUPSI (Berufsuniversität der italienischen Schweiz) hat eine Reihe technischer Vorschläge formuliert, um die Sicherheit von Personen in ihrem Zuhause zu erhöhen. Was waren die Ziele des Projekts IDA-GRANNO, und wie gestaltet die SUPSI ihre Vorgehensweise?
\end{abstract}

Laura Bertini: IDA-GRANNO war ein stark interdisziplinär geprägtes und komplexes Projekt. Ziel der Arbeitsgruppe war es, die Autonomie im häuslichen Bereich zu unterstützen und das Gefühl der Sicherheit zu stärken, und zwar durch ein Bündel an Gesundheitstechnologien, die an den Benutzer angepasst werden können. Durch die Programmierung von Alltagsgegenständen ermöglichte es das System, bestimmte alltägliche Aufgaben zu automatisieren und sicher zu gestalten. Motor des Projekts war die Sturzmeldefunktion, eine sehr komplizierte Entwicklungsaufgabe und als solche eine Herausforderung, die sich als zu ambitioniert erwies. Das Ziel war, im Rahmen des besagten Technologienbündels eine Uhr zu entwickeln, die in der Lage sein würde, Stürze zu erkennen und einen passiven Alarm an Angehörige oder Pflegedienste auszugeben. Damit sollten die Folgen solcher Vorkommnisse, die besonders mit fortschreitendem Alter verbunden sind, abgemildert werden.

Stürze werden also als Kostenfaktor und Ursache von Abhängigkeit gesehen. Diese Einordnung kritisieren Sie - warum?

In der Epidemiologie wurde das Alter als Risikofaktor für Osteoporose definiert. Von daher sind Stürze ein Thema für das Gesundheitswesen geworden. In diesem Zusammenhang sind bestimmte Assoziationen verbreitet: Wenn „Stürze bei Senioren“ angesprochen werden, gibt es so etwas wie einen allgemeinen Konsens darüber, dass ein solches Vorkommnis eine ganze Reihe von Problemen auslösen kann, darunter auch einen Verlust an Autonomie. Jedoch ist es sehr schwierig, einen Sturz eindeutig zu definieren: Mit diesem Begriff bezeichnet man eine Vielzahl an sehr unterschiedlichen Situationen - und muss daher sehr aufpassen, wenn man bei der Arbeit an technischen Lösungen für den sozialen Bereich von Stürzen spricht. Personen stürzen nicht auf die gleiche Art, und vor allem lassen nicht alle Stürze dieselbe Interpretation zu. Ein Sturz kann auch eine quasi symbolische Funktion haben, indem er den Übergang ins Alter kennzeichnet und damit für Kontrollverlust steht. Die Angst zu stürzen, die auch einen Risikofaktor darstellt, wurde von gebrechlichen Senioren, die an entsprechenden Projekten beteiligt waren, als Angst definiert, nicht wieder aufstehen zu können. Hier wendet sich das Problem, und die sozialtechnische Lösung kann nicht mehr auf die Erkennung eines stürzenden Körpers abzielen, der mit einem automatischen, von Benutzer unabhängigen Alarm verbunden ist - eine Funktion, die 
unterstützende Angehörige beruhigt. Und schliesslich ist es auch so, dass gar kein Alarm ausgelöst wird, wenn man nicht wie jüngere Menschen in einem Labortest stürzt, die sich auf eine Matratze fallen liessen. Personen, die das Bedürfnis nach Absicherung haben, möchten Alarm auf einfache, autonome Weise auslösen, weil es ja nicht nur um einen Körper geht, der hinfällt. Und wenn man mal den Aspekt der medizinischen Betreuung auslässt und die Perspektive des Benutzers einnimmt, verändern die technisch umzusetzenden Bedürfnisse den Blick auf die Gegenstände und ihre Funktionen.

Senioren sehen sich also mit „Präventionsmitteln“ für Probleme konfrontiert, die sie nicht als ihre eigenen empfinden. Die als ,, Rettungsanker" dargestellte Technologie wird in ihren Augen dann bedeutungslos. Wenn wir mal von dem Projekt ausgehen, welche kritischen Anmerkungen hätten Sie dann zur Einführung technischer Mittel zur Unterstützung von Senioren und der Pflege, die sie womöglich benötigen?

Ob Senioren oder nicht - Menschen, die Schwierigkeiten bei der Ausführung einer Alltagsaktivität haben, entwickeln unterschiedliche Kompensationsstrategien. Denn das Lebensumfeld ist nicht nur ein objektiver Raum, sondern vor allem ein Raum mit subjektiven Bezugspunkten für unser Dasein. Wenn man nun eine Veränderung vornimmt, z. B. eine technische Vorrichtung einführt, kann uns diese entweder dabei helfen, eine Aktivität auf effektivere Weise auszuführen - oder aber uns durch die damit verbundene Umstrukturierung unserer Umgebung entfremden. So war in einem Beispiel ein Medikamentenspender darauf programmiert, den Benutzer (durch ein akustisches Signal) und danach die Angehörigen zu alarmieren, falls der Benutzer vergessen hatte, zur festgesetzten Zeit das Medikament einzunehmen. Der Benutzer, der das Medikament zuvor anhand eines Signals beim Mittagsjournal im Radio eingenommen hatte, liess sich nun von der neuen Vorrichtung dazu veranlassen. Die vorgesehene Nutzung, d. h. die Kontrolle der Medikamenteneinnahme und - falls diese vergessen worden war - eine entsprechende Massnahme, war somit nicht sehr kompatibel mit dem Interesse des Benutzers, sein Medikament nicht zu vergessen.

Die Unterschiedlichkeit von Senioren und die subjektiven Aspekte des Lebens sind wahrscheinlich die am häufigsten übersehenen Gesichtspunkte bei technologischen Ansätzen, mit denen Lösungen für die Probleme einer wachsenden Anzahl von Senioren in der Gesellschaft angestrebt werden. Gibt es also keinen Raum für technische Beiträge in unserem Bereich?

Senioren verfügen wie alle Menschen über grossen Einfallsreichtum und ein hohes Mass an Anpassungsfähigkeit an die teils schwierigen Bedingungen des täglichen Lebens. Ob in fortgeschrittenem Alter oder nicht - die Benutzer einer technischen Vorrichtung machen sich mit dieser vertraut, wenn sie das Gefühl haben, dass es ihnen diese Vorrichtung ermöglicht, objektiv und auch subjektiv mit ihrem Lebensumfeld etwas besser zurechtzukommen. Anders ausgedrückt: Wir alle schätzen es, effektiver zu sein, wenn wir irgendein Hilfsmittel übernehmen - andernfalls lassen wir es genauso schnell wieder fallen. Jede technische Lösung muss auf einem Bedürfnis basieren, das vom Benutzer klar definiert 
wurde, und seinen Anforderungen in puncto Anwendbarkeit entsprechen - d. h. die entsprechende Vorrichtung muss von einer Person in Bezug auf ihren Körper, ihre Umgebung und ihre Wohnsituation gut zu handhaben sein.

Dr. Laura Bertini ist Anthropologin und Forscherin am Centro competenze anziani (Senioren-Kompetenzzentrum) der Berufsuniversität der italienischen Schweiz.

Kontakt: laura.bertini@supsi.ch

Stefan Spring ist Forschungsbeauftragter am Schweizerischen Zentralverein für das Blindenwesen (SZB). Kontakt: spring@szb.ch 